Human Papillomavirus (HPV-16/18) AS04-adjuvanted vaccine (Cervarix ${ }^{\circledR}$ ) on the serum and cervicovaginal microenvironment, characterizing the $\mathrm{TH} 1 / \mathrm{TH} 2$ cytokines profile.

Methods A subset of 20 women between 18 and 40 years old without genital coinfections (bacterial vaginosis, Herpes virus, Candida sp Neisseira gonorrhea or Chlamydia trachomatis) were selected to receive the three doses of the HPV-16/18 AS04-adjuvanted vaccine $\left(\right.$ Cervarix ${ }^{\circledR}$ ). Blood and cervicovaginal samples were collected before the first dose and 30 days after the third dose. TH1 (INF- $\gamma$, IL2, IL-12p70, TNF- $\alpha$, GM-CSF) and TH2 (IL-4, IL-5, IL-10, IL-13) cytokines were determined by Immunology Multiplex.

Results In the blood samples there were no statistically significant differences in the level of cytokines before or after the three doses of the vaccine, except for TNF- $\alpha$ (INF- $\gamma$ : $\mathrm{p}=0.797 ; \quad$ IL2: $\mathrm{p}=0.735, \quad$ IL-12p70: $\mathrm{p}=0.881 ;$ TNF- $\alpha$ : $\mathrm{p}=0.011$, GM-CSF, $\mathrm{p}=0.721 ; \mathrm{IL}-4, \mathrm{p}=0.223 ; \mathrm{IL}-5, \mathrm{p}=0.860$; IL-10, $p=0.473$; IL-13, $p=0,913$.). However, for the CVC samples, there was a tendency to decrease the cytokine level after the three doses of the vaccine. This decrease was significant for the INF- $\gamma(p=0,010), \quad$ IL-5, $(p=0,005)$, IL-12p70 $(\mathrm{p}=0,002)$ e IL-13 ( $\mathrm{p}=0,002)$.

Conclusion TH1/TH2 cytokines were detected in serum of women who received the HPV-16/18 AS04-adjuvanted vaccine, but there were no significant differences before and after the three doses. In the vaginal samples there was a significant decrease of INF- $\gamma$, IL-12p70, IL-5 and IL-13. Understanding the clinical significance of these modifications is a very relevant issue and future studies that address the network of inflammatory and anti-inflammatory cytokine effects should be considered.

Disclosure No significant relationships.

\section{P838 ASSOCIATIONS OF THE VAGINAL MICROBIOTA WITH HPV INFECTION AND CERVICAL DYSPLASIA IN SOUTH AFRICAN WOMEN LIVING WITH HIV}

${ }^{1} J a n n e k e$ Van De Wijgert*, 'Allessandra Gill, ${ }^{1}$ Alistair Darby, ${ }^{2}$ Helen Kelly, ${ }^{3}$ Admire Chikandiwa, ${ }^{4}$ Sinead Delany-Moretlwe, ${ }^{5}$ Marijn Verwijs, ${ }^{6}$ Suzanna Francis, ${ }^{6}$ Philippe Mayaud. 'University of Liverpool, Liverpool, UK; ${ }^{2}$ London School of Hygiene and Tropical Medicine, London, UK; ${ }^{3}$ Wits RHI, University of the Witwatersrand, Johannesburg, South Africa; ${ }^{4}$ University of the Witwatersrand, Wits RHI, Johannesburg, South Africa; ${ }^{5}$ University of Liverpool, Institute of Infection and Global Health, Liverpool, UK; ${ }^{6}$ London School of Hygiene and Tropical Medicine, London, UK

\subsection{6/sextrans-2019-sti.883}

Background Fifteen longitudinal studies have shown associations between bacterial vaginosis and high risk human papillomavirus (hrHPV) acquisition and/or persistence, and/or cervical dysplasia. However, few studies assessed the vaginal microbiota (VMB) comprehensively, and none controlled the dysplasia association for persistent hrHPV.

Methods 623 women attending HIV outpatient clinics in Johannesburg, South Africa, were examined for hrHPV (InnoLipA HPV Genotyping Extra Assay), cervical dysplasia (histology), and vaginal microbiota (VMB; V3-V4 Illumina HiSeq 2x300bp with Swarm OTU-picking) at baseline and endline, a median of 16 months after baseline. VMB research questions were addressed in two nested case-control designs.

Results Hierarchical clustering resulted in seven VMB types: $L$. iners-dominated ( $\mathrm{Li} ; \mathrm{n}=214$ samples), Lactobacillus crispatus or L. jensenii-dominated (Lcj; $\mathrm{n}=68)$, Bifidobacterium-dominated (BD; $\mathrm{n}=2)$, lactobacilli + bacterial vaginosis (BV)- anaerobes $(\mathrm{L}+\mathrm{A} ; \mathrm{n}=208)$, BV-like (BV; $\mathrm{n}=303)$; BV-anaerobe dominated $(\mathrm{AD} ; \mathrm{n}=56)$; and pathobiont-characterised $(\mathrm{PB}$; $\mathrm{n}=19$ ). Women with new or persistent hrHPV during followup were less likely to have an Lcj VMB type (compared to Li) at endline, and persistent hrHPV was associated with vaginal anaerobic dysbiosis at baseline (decreased lactobacilli, increased BV-anaerobes, and increased Nugent score). Women who developed CIN2+, compared to women with persistent hrHPV but no CIN2+, were more likely to have vaginal anaerobic dysbiosis at endline (decreased lactobacilli, increased BV-anaerobes, and increased diversity), but not at baseline. These associations persisted after controlling for age, hormonal contraception, and CD4+ count; several additional potential confounders (HIV plasma viral load, antiretroviral therapy, sexually transmitted infections, sexual risk-taking, among others) were evaluated.

Conclusion Frequent hrHPV exposure (and/or increased sexual risk-taking) likely causes vaginal dysbiosis, but a bilateral relationship cannot be ruled out. Women with vaginal dysbiosis are not at increased risk of CIN2+ development when hrHPV status is taken into account, but vaginal dysbiosis does develop when CIN2+ lesions develop. These results should be confirmed in even larger longitudinal studies.

Disclosure No significant relationships.

\section{P839 A CHEMICALLY MODIFIED $\beta$-LACTOGLOBULIN (JB01) IS EFFECTIVE IN TREATING HPV INFECTION AND PREVENTING SEXUAL TRANSMISSION OF HIV}

Shibo Jiang*. Fudan University, School of Basic Medical Sciences, Shanghai, China

\subsection{6/sextrans-2019-sti.884}

Background More than $90 \%$ of the new HIV infection in China occurred through sexual transmission, particularly among the men who have sex with men (MSM). Cervical cancer, the second most common cancer among women, is caused by sexually acquired infection with high-risk types of HPV, such as types 16 and 18 . We previously have shown that a chemically modified bovine milk protein, $\beta$-lactoglobulin (3HP$\beta$-LG, also known as JB01), is effective against infection by a broad-spectrum of HIV (Nat. Med., 2:230,1996) and HPV entry inhibitor. Therefore, we intended to develop topical formulations containing JB01 against HIV and HPV infections.

Methods A pseudotyped HPV particles expressing HPV L1 and L2 proteins were used for testing the inhibitory activity of JB01. A randomized open-label clinical trial of a JB01 biological dressing (JB01-BD) administered intravaginally was performed to evaluate its in vivo safety and efficacy. Both pseudotyped and live HIV-1 strains with different subtypes and tropisms were used for evaluating the in vitro efficacy of JB01 and a non-human primate (NHP) model was used for testing the in vivo efficacy of JB01.

Results The trial of JB01-BD administered intravaginally demonstrated that JB01-BD is safe and effective. About $60.5 \%$ and $13.5 \% \mathrm{HPV}$-positive women in the treatment and nontreatment groups, respectively, became HPV-negative $(P<$ 0.001). In vitro study suggests that JB01 exhibits broad-spectrum antiviral activity against divergent HIV-1 strains, including those resistant to the current antiretroviral therapeutics. Rhesus macaques monkeys pretreated with a topical formulation were protected against rectal challenge with SHIVSF162P3. 
Conclusion JB01-BD is safe and effective against HPV and HIV infections, and was approved by China Food and Drug Administration (CFDA) for treating cervical HPV infection with the potential to reduce morbidity of cervical cancer. A lubricant formulation containing JB01 has recently been approved by CFDA as an auxiliary strategy to prevent sexual transmission of HIV.

Disclosure No significant relationships.

\section{P840 THE HPV SCREENING AND VACCINE EVALUATION (HPV- SAVE) STUDY IN MEN LIVING WITH HIV: EARLY PATHOLOGIC AND ACCEPTABILITY OUTCOMES}

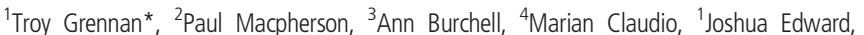
${ }^{3}$ Jennifer Gillis, ${ }^{5}$ Daniel Grace, ${ }^{6}$ Ronita Nath, ${ }^{4}$ Ron Rosenes, ${ }^{7}$ Darrell Tan, ${ }^{4}$ Irving Salit. ${ }^{1} B C$ Centre for Disease Control, Clinical Prevention Services, Vancouver, Canada; ${ }^{2}$ The Ottawa Hospital, Ottawa, Canada; ${ }^{3}$ St. Michael's Hospital, Li Ka Shing Knowledge Institute, Toronto, Canada; ${ }^{4}$ University Health Network, Toronto, Canada; ${ }^{5}$ University of Toronto, Dalla Lana School of Public Health, Toronto, Canada; ${ }^{6}$ British Columbia Centre for Disease Control, Clinical Prevention, Vancouver, Canada; ' 5 t. Michael's Hospital, Toronto, Canada
\end{abstract}

\subsection{6/sextrans-2019-sti.885}

Background Anal cancer caused by oncogenic, high-risk (HR) human papillomavirus (HPV) is emerging as a leading cause of non-HIV-related death in HIV-positive MSM. Anal cancer rates in HIV-positive MSM are up to 100-times higher than the general population. There are no universally-accepted guidelines for anal cancer screening, even in high risk populations, due to a paucity of evidence to support its effectiveness. We assessed the acceptance rate to invitations for anal cancer screening, and describe preliminary pathology results.

Methods The HPV-SAVE Study is an ongoing Canadian study on screening and treatment of anal cancers and pre-cancers in HIV-positive MSM. Participants were invited to have anal cytology and HPV testing in their physician's office. Those with abnormalities were referred for high resolution anoscopy (HRA) and anal biopsies. Cytology was graded as per the Bethesda classification, and histology was described per the Lower Anogenital Squamous Terminology (LAST) nomenclature.

Results Out of 2241 invitations as of 01/2019, 617 men $(27.5 \%)$ agreed to be screened. Cytology results from 518 satisfactory Pap tests were: 246 negative (47.5\%), 62 LSIL (12.0\%), 9 LSIL-H (1.7\%), 14 HSIL (2.7\%), 174 ASCUS (33.6\%), and 13 ASC-H (2.5\%). In 116 participants referred for HRA, 247 biopsies were done, yielding HSIL in 62 $(53.4 \%)$ unique individuals, and one invasive carcinoma. In a sample of 127 participants, $111(87.4 \%)$ had any HPV type, $82(64.6 \%)$ had multiple HPV types, 78 (61.4\%) had at least one high-risk HPV type, and 39 (30.7\%) had HPV-16.

Conclusion MSM living with HIV had moderate acceptance of anal cancer screening invitations, with over half of screened men having abnormal cytology. A majority of those undergoing HRA had high-grade histology diagnosed, and most participants had HPV anal canal infection, with nearly two-thirds having anal canal infection with HR-HPV. These early results highlight the enormous HPV burden in this high-risk population.

Disclosure No significant relationships.

\section{P841 BARRIERS TO HPV VACCINATION AMONG GAY, BISEXUAL, AND OTHER MEN WHO HAVE SEX WITH MEN (GBMSM) IN CANADA: A CIRN STUDY}

${ }^{1}$ Ramandip Grewal ${ }^{*},{ }^{1}$ Anna Yeung, ${ }^{2}$ Marc Brisson, ${ }^{3}$ Troy Grennan, ${ }^{4}$ Alexandra De Pokomandy, ${ }^{4}$ Joseph Cox, ${ }^{5}$ Gilles Lambert, ${ }^{6}$ David Moore, ${ }^{7}$ François Coutlée, ${ }^{8}$ Shelley Deeks, ${ }^{9}$ Sandra Gardner, ${ }^{10}$ Dane Griffiths, ${ }^{11}$ Wanrudee Isaranuwatchai, ${ }^{12}$ Jody Jollimore, ${ }^{13}$ James Murray, ${ }^{14}$ Gina Ogilvie, ${ }^{15}$ Chantal Sauvageau, ${ }^{1}$ Darrell Tan, ${ }^{16}$ Barry Adam, ${ }^{14}$ Heather Armstrong, ${ }^{9}$ Mark Gaspar, ${ }^{17}$ Clemon George, ${ }^{18}$ Daniel Grace, ${ }^{19}$ Trevor Hart, ${ }^{1}$ Ann Burchell. 'St. Michael's Hospital, Centre For Urban Health Solutions, Li Ka Shing Knowledge Institute, Toronto, Canada; ' Université Laval, Quebec City, Canada; ${ }^{3} B C$ Centre for Disease Control, Clinical Prevention Services, Vancouver, Canada; ${ }^{4}$ McGill University, Montreal, Canada; ${ }^{5}$ University of Montreal, Montreal, Canada; ${ }^{6} B C$ Centre for Excellence in HIVIAIDS, Vancouver, Canada; 'I'Université de Montréal, Microbiologie Médicale et Infectiologie, Montreal, Canada; ${ }^{8}$ Public Health Ontario, Toronto, Canada; ${ }^{9}$ University of Toronto, Toronto, Canada; ${ }^{10}$ Gay Men's Sexual Health Alliance, Toronto, Canada; ${ }^{11}$ St. Michael's Hospital, Toronto, Canada; ${ }^{12}$ Community Based Research Centre, Vancouver, Canada; ${ }^{13}$ Ontario Ministry of Health and Long-Term Care, Toronto, Canada; ${ }^{14}$ University of British Columbia, Vancouver, Canada; ${ }^{15}$ Institut National de Santé Publique du Québec, Montreal, Canada; ${ }^{16}$ University of Windsor, Windsor, Canada; ${ }^{17}$ University of West Indies - Cave Hill, Cave Hill, Barbados; ${ }^{18}$ University of Toronto, Dalla Lana School of Public Health, Toronto, Canada; ${ }^{19}$ Ryerson University, Psychology, Toronto, Canada

\subsection{6/sextrans-2019-sti.886}

Background Internationally, Canada was one of the first countries to offer free HPV vaccination to young gbMSM. The provinces of British Columbia (BC), Ontario (ON) and Quebec (QC) implemented HPV vaccination programs in 20152016 for gbMSM aged 9-26. We explored HPV vaccine initiation and among unvaccinated men, potential barriers to uptake.

Methods Engage is a sexual health study among gbMSM aged $16+$ in Vancouver BC, Toronto ON, and Montreal QC, the largest urban centres in each province. Men are recruited via respondent driven sampling (RDS). We estimated the proportion that had initiated HPV vaccination and among unvaccinated men, compared proportions to responses on HPV vaccine knowledge and willingness, healthcare access and sexual orientation disclosure by age (eligible for free vaccination: $\leq 26$ and ineligible for free vaccination: $\geq 26$ years old). Proportions were RDS unadjusted.

Results From 01/2017 to 31/12/2018, 2099 men enrolled (542 Vancouver, 378 Toronto, 1179 Montreal). Their median age was 33 years (IQR 27-46). In Vancouver, Toronto, and Montreal, $48.6 \%, 44.1 \%$ and $44.2 \%$ of men aged $\leq 26$ and $24.9 \%$, $30.3 \%$ and $7.1 \%$ of men aged $\geq 26$ had $1+$ dose of the HPV vaccine, respectively. Among unvaccinated men, more men aged $\geq 26$ versus $\leq 26$ accessed healthcare (family doctor/sexual health/HIV care) $(87.2 \%$ vs $78.9 \%, \mathrm{p}=0.0008)$ and disclosed their sexual orientation to their family doctor $(86.2 \%$ vs $64.4 \%, \mathrm{p}<0.0001)$. A similar proportion of unvaccinated men from each age group had heard of the HPV vaccine $(\geq 26=67.5 \%$ vs $\leq 26=69.0 \%, p<0.0529)$. Among unvaccinated men aware of the HPV vaccine, more men aged $\geq 26$ versus $\leq 26$ were willingness to get vaccinated if the vaccine were free and required disclosure of same-sex activity $(80.3 \%$ vs $61.5 \%, \mathrm{p}=0.0259)$.

Conclusion Preliminary analyses indicate that HPV vaccine initiation in this population remains suboptimal. Among unvaccinated gbMSM $\leq 26$, accessing healthcare, not disclosing sexual orientation and willingness to get vaccinated may be barriers to vaccine uptake.

Disclosure No significant relationships. 